\title{
Integración de sistema SCADA y arquitectura distribuida para la automatización de un proceso de llenado de contenedores y molienda de grano título principal
}

\author{
Integration of SCADA system and distributed architecture for the automation of a process of \\ filling grain containers and grain milling
}

\author{
E. Flores-García ${ }^{a}$, J.C. Quezada-Quezada ${ }^{b}$, J. Tapia-Suárez $^{c}$, \\ R. Casiano-Rivera ${ }^{d}$, R.V.H. Calderón-Medina ${ }^{e}$
}

\begin{abstract}
:
This work deals with a project proposal focused on the communication between two different brand controllers, such as the PLC Festo Compact FC34 and the GE PACSystem RX3i. For the considered application, each controller executes a different process being interrelated through a distributed architecture; likewise, data acquisition is carried out by means of the supervision software Wonderware InTouch, which in this case is also used for accomplishing a system remote monitoring, and by using a router the information collected from the controllers is concentrated and wirelessly transmitted. Both the PLC Festo Compact and the GE PACSystem RX3i have been developed a local Human-Machine Interface, with the purpose of providing the field operator the facility to monitor and control the process when required. The proposal has been implemented through an industrial-type application focused to controlling a process of filling a couple of grain containers, as well as of grain transporting and grinding. Variables related to application physical inputs and outputs (sensors, valves, motors, etc.) are simulation-level generated signals, with the aim of demonstrating the system operability proposed in the project.
\end{abstract}

Keywords:

HMI, Industrial process, PAC, PLC, SCADA

\section{Resumen:}

En este trabajo se presenta la propuesta de un proyecto enfocado a la comunicación entre dos controladores de marca distinta, como son el PLC Festo Compact FC34 y el GE PACSystem RX3i. Para la aplicación considerada, cada controlador ejecuta un proceso distinto pero que están interrelacionados mediante una arquitectura distribuida; a su vez, la adquisición de datos se lleva a cabo a través del software de supervisión Wonderware InTouch que, en este caso, se utiliza también para realizar un monitoreo remoto del sistema; y empleando un router, la información recabada de los controladores se concentra y se transmite de manera inalámbrica. Tanto al PLC Festo Compact como al GE PACSystem se le ha desarrollado a cada uno una Interfaz Humano-Maquina local, con el propósito de proporcionar al operador en campo la facilidad de monitorear y controlar el proceso cuando sea requerido. La propuesta ha sido implementada a través de una aplicación de tipo industrial enfocada al control de un proceso de llenado de un par de silos contenedores de grano, así como de transporte y molienda del grano. Las variables relacionadas con las entradas y salidas físicas de la aplicación (sensores, válvulas, motores, etc.) son señales generadas a nivel de simulación, con la finalidad de demostrar la operatividad del sistema propuesto en el proyecto.

\section{Palabras Clave:}

HMI, PAC, PLC, Proceso industrial, SCADA

\section{Introducción}

En la actualidad existen industrias cuyos procesos presentan tal complejidad que requieren ser divididos en subprocesos que permitan un mejor control para cada uno de ellos; sin embargo la automatización de dichos procesos no siempre se logra de manera conjunta lo cual, dada la creciente oferta en el mercado de los controladores y de la instrumentación, como consecuencia de la acelerada actualización e innovación tecnológica, origina que existan subprocesos que, a pesar de estar interrelacionados en la cadena de producción, 
deban ser controlados de manera independiente por medio de dispositivos y sistemas de marcas y modelos diferentes, generando la necesidad y el surgimiento del control distribuido.

El presente trabajo pretende responder a tal problemática, planteando una propuesta de alternativa de solución para la interconexión entre dispositivos de control de distinta marca, permitiendo su comunicación e interoperabilidad a través de un sistema supervisorio único que sirva como enlace entre los distintos controladores de los subprocesos relacionados.

\section{EI Modelo OSI}

El modelo de interconexión de sistemas abiertos, OSI (por sus siglas en Inglés de Open System Interconnection), se divide en 7 capas, donde una primera parte, constituida por los niveles 5, 6 y 7, se encuentra orientada a dar soporte al usuario; la parte restante la componen los niveles 1, 2, 3 y 4, y abarca temas referentes al flujo de información digital entre terminales y maquinas. Este modelo se toma como base para la conexión entre equipos mediante reglas y protocolos para la trasmisión y recepción de información [1].

\section{Sistema SCADA (Supervisory Control and Data Acquisition)}

Un sistema SCADA (por sus siglas en Inglés de Supervisory Control And Data Acquisition; Control Supervisorio y Adquisición de Datos), es una aplicación de software diseñada con el propósito de controlar y supervisar procesos a distancia, por medio de Interfases de usuario [2].

El software de tipo supervisorio se basa en la adquisición de datos de procesos remotos (a distancia) obteniendo la recolección de datos históricos, informes de los instrumentos en campo y está diseñado primordialmente para trabajar sobre ordenadores en el control de producción, realizando el control de procesos de forma automática desde un ordenador central (supervisor) y facilitando la comunicación con los dispositivos de campo, como son los Controladores de Automatización Programables (PAC, por sus siglas en Inglés de Programmable Automation Controller), Controladores Lógicos Programables (PLC, por sus siglas en Inglés de Programmable Logic Controller), instrumentos de medición, y actuadores o elementos finales de control.

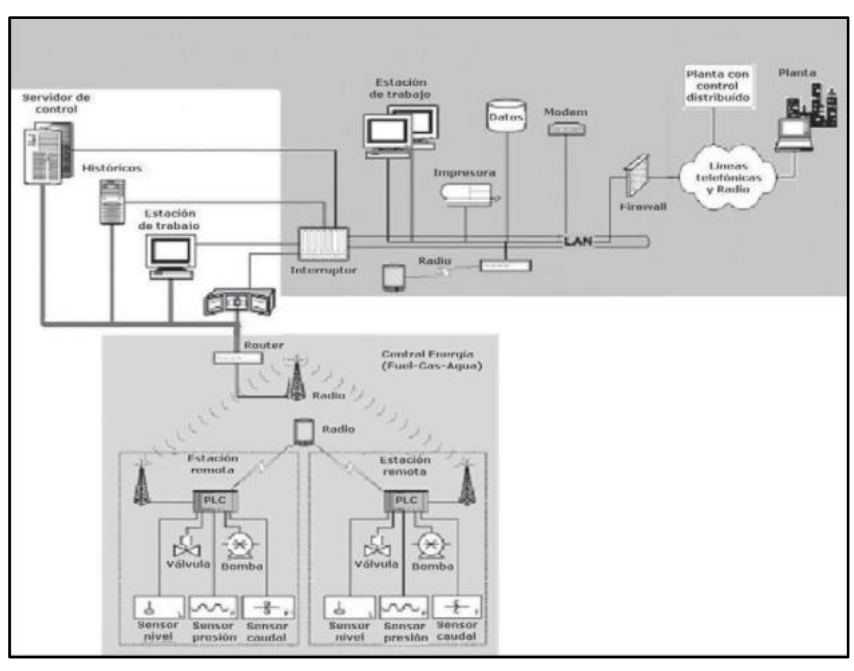

Figura 1. Control SCADA [3].

En los últimos años, los sistemas SCADA han permitido progresos importantes en términos de funcionalidad, rendimiento y escalabilidad, los cuales representan una alternativa para los muy exigentes y complejos sistemas de control inmersos en los procesos industriales [4].

\section{Pirámide CIM}

En la industria se utilizan distintas redes de comunicación a diferentes niveles del diseño de la Pirámide CIM (por sus siglas en Inglés de Computer Integrated Manufacturing; Manufactura Integrada por Computadora). En este modelo, cada nivel efectúa una tarea específica, en el cual tiene una información y un procesamiento distinto asociada a ella.

La pirámide CIM se describe como una filosofía y estrategia de producción, caracterizada por integrar toda la información de las diferentes áreas de una empresa por medio de sistemas informáticos y del uso de equipos electrónicos para el control, supervisión y gestión de los procesos [5].

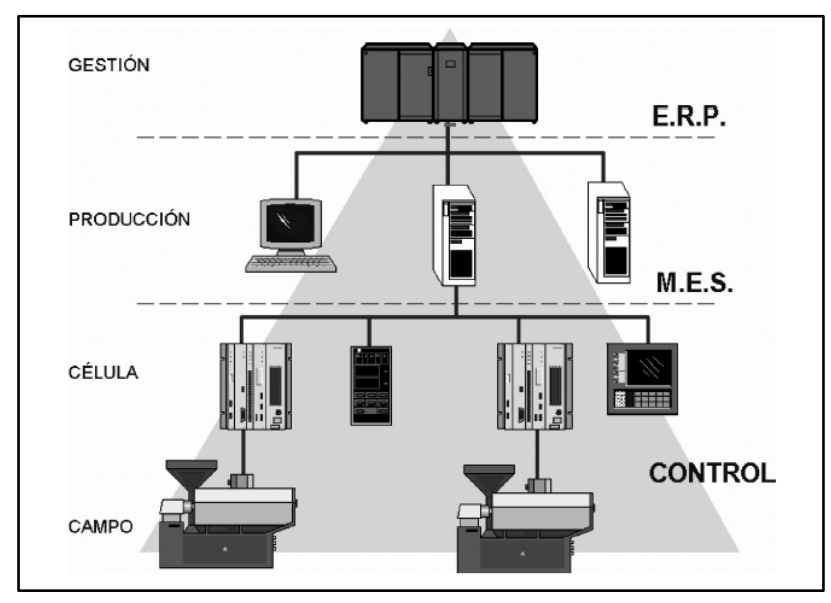

Figura 2. Pirámide CIM [2]. 


\section{Comunicación}

La comunicación y el enlace entre ambos controladores se realiza por medio del protocolo de comunicación Ethernet, para realizar la comunicación entre el PLC y el sistema SCADA; existen varios sistemas como el OPC (por sus siglas en Inglés de OLE for Process Control; a su vez OLE de Object Linking and Embedding; enlace e incrustación de objetos para control de procesos) y el DDE (por sus siglas en Inglés de Dynamic Data Exchange; Intercambio Dinámico de Datos); en el cual se hace uso de ambos para realizar las comunicaciones.

Una aplicación DDE y OPC puede comportarse como:

- Cliente: solicita datos a un servidor.
- Servidor: proporciona datos a un
cliente.
Cliente/Servidor: solicita y proporciona
información.
Monitor: puede acceder a mensajes sin
modificarlos.

Un servidor OPC es un software (driver) que cumple con uno o varios datos específicos definidos por la Fundación OPC. Los Servidores OPC hacen las veces de interfaz, por un lado, comunicando una o varias fuentes de datos que usan sus protocolos natales (PLCs, DCSs, módulos, reguladores, entre otros); por otro, ponerse del lado de Clientes OPC (SCADAs, HMls, generadores de gráficos, entre otros). EI OPC se caracteriza por ser invariable, por tratar objetos distintos de la misma forma, a lo que se le denomina polimorfismo, por ser implementadas por servidores OPC y por conectar objetos, de tal modo que éstos y sus clientes pueden establecer una comunicación bidireccional [3], lo que significa que los Clientes pueden leer y escribir a los dispositivos por el Servidor OPC [6].

\section{Protocolo Ethernet}

Para soluciones eficientes de automatización con Industrial Ethernet, el ámbito industrial, dispone de una potente red de área y célula según el estándar IEEE 802.3 (Ethernet) y 802.11 (Wireless LAN). Actualmente Ethernet es, con una proporción de más del $80 \%$, el número uno en todo el mundo entre las redes LAN. Este sistema permite crear, con Industrial Ethernet, potentes redes de comunicación de gran extensión" [7]. Las múltiples posibilidades de Intranet, Extranet e Internet que ya están disponibles actualmente en el ámbito ofimático, también se pueden aprovechar en la automatización de procesos.

\section{Controlador Lógico Programable, PLC}

Se entiende por PLC, o Autómata Programable, a aquel dispositivo electrónico diseñado para controlar, en tiempo real y en entornos industriales, procesos secuenciales. Su manipulación y programación puede ser realizada por personal del ramo eléctrico o electrónico, incluso sin conocimientos informáticos [8].

La norma IEC 61131-1 [9], menciona que un PLC es un sistema electrónico que opera digitalmente, diseñado para uso en ambiente industrial; tiene una memoria de almacenamiento programable, para almacenar instrucciones establecidas por el usuario para funciones específicas como la secuencia, la lógica, y la aritmética; para controlar, a partir de entradas/salidas digitales $\mathrm{y} / \mathrm{o}$ analógicas, varios tipos de máquinas o procesos; también puede tomar decisiones con base en criterios preprogramados, y comunicarse con otros sistemas externos. Los PLC se consideran miembros de la familia de circuitos de estado sólido, llegando a reemplazar a los dispositivos electromecánicos utilizados para implementar funciones de control.

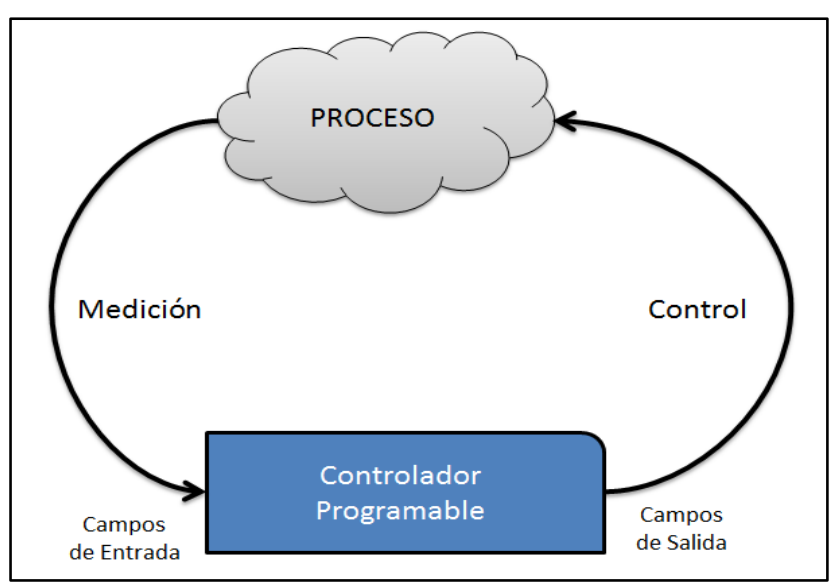

Figura 3. Diagrama conceptual de una aplicación de PLC [8].

\section{Softwares empleados en la implementación del sistema SCADA}

\section{Software SCADA Wonderware InTouch}

Para realizar acciones de monitoreo y control en un sistema, se requiere un software SCADA para la creación de pantallas de HMI (por sus siglas en Inglés de HumanMachine Interface; Interfaz Humano-Máquina) y de bases de datos; para ello, en el presente trabajo se empleó Wonderware InTouch, software de tipo supervisorio que ofrece herramientas para crear aplicaciones de HMI para Microsoft Windows. Además, InTouch proporciona funciones de control y monitoreo de procesos industriales. "Mediante InTouch, se puede crear aplicaciones potentes y con todas las funciones que explotan las características clave de Microsoft Windows, incluidos los controles ActiveX, OLE, gráficos, redes, y más" [10]. InTouch también se puede ampliar agregando controles ActiveX personalizados, asistentes, objetos genéricos y creando extensiones InTouch QuickScript.

\section{Servidores para la comunicación}


En este apartado se comenta sobre algunas especiaciones del servidor OPC KepServer y del servidor IPC Server de Festo, los cuales se emplearon para la comunicación de los PLC con el software SCADA Wonderware InTouch.

OPC KepServer es un servidor basado en software que está diseñado para comunicaciones precisas, configuración rápida y una interoperabilidad excepcional entre aplicaciones cliente, dispositivos industriales y sistemas. El servidor ofrece una amplia gama de complementos y controladores de dispositivos y componentes que se adaptan a la mayoría de las necesidades de comunicación [11].

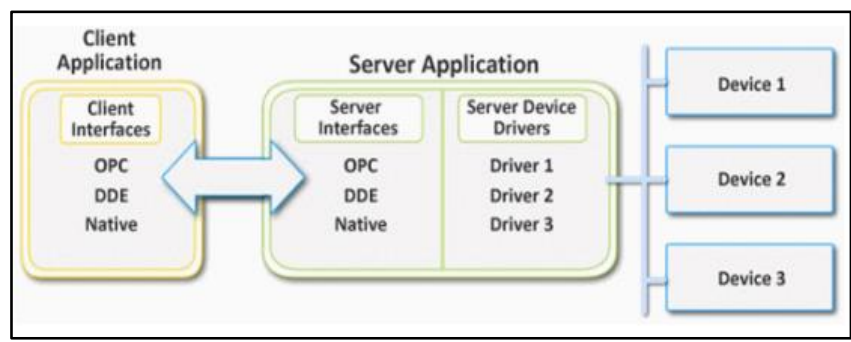

Figura 4. Forma de comunicación del servidor KepServer con los controladores [11].

El Servidor de Comunicaciones IPC de FESTO (denominado de aquí en adelante "Servidor IPC FESTO" o "FESTO IPC" o simplemente "Servidor"), es un programa de aplicación de Microsoft Windows que actúa como servidor de protocolos de comunicación y permite a otros programas de aplicación de Windows acceder a datos de FESTO PCs industriales FST (IPC), por ejemplo FEC FC34-FST, utilizando comunicaciones TCP/IP. EI servidor FESTO IPC requiere una tarjeta Ethernet y un protocolo TCP/IP instalados en el equipo para comunicarse con los IPC de FESTO a través de la red Ethernet [12].

\section{Descripción del Sistema}

El presente trabajo, como parte del desarrollo del proyecto propuesto, se enfoca en la comunicación de dos controladores de marca distinta, como son el PLC Festo Compact FC34 y el GE PACSystem RX3i; en la aplicación considerada, cada controlador ejecuta un proceso distinto, pero que están interrelacionados mediante una arquitectura distribuida; a su vez, la adquisición de datos se lleva a cabo a través del software supervisorio Wonderware InTouch que, en este caso, es utilizado también para realizar un monitoreo remoto del sistema; y empleando un router, la información colectada de los controladores es concentrada y transmitida de manera inalámbrica. El software Wonderware InTouch, dentro de sus funciones, sirve como enlace o interfaz para que ambos dispositivos puedan comunicarse entre sí, y tendrá la capacidad de tomar acciones de control que permitan la regulación del proceso, y a su vez una base de datos de la información obtenida desde los controladores sobre el estado y control de los procesos.

La ventaja del uso de un control de tipo distribuido es que permite que cada controlador esté encargado de procesos distintos en paralelo, ayudando a incrementar la confiabilidad de la estructura del sistema, y permitiendo una mayor seguridad, economía de funcionamiento, y brindando la facilidad de ampliar o modificar los procesos de la planta.

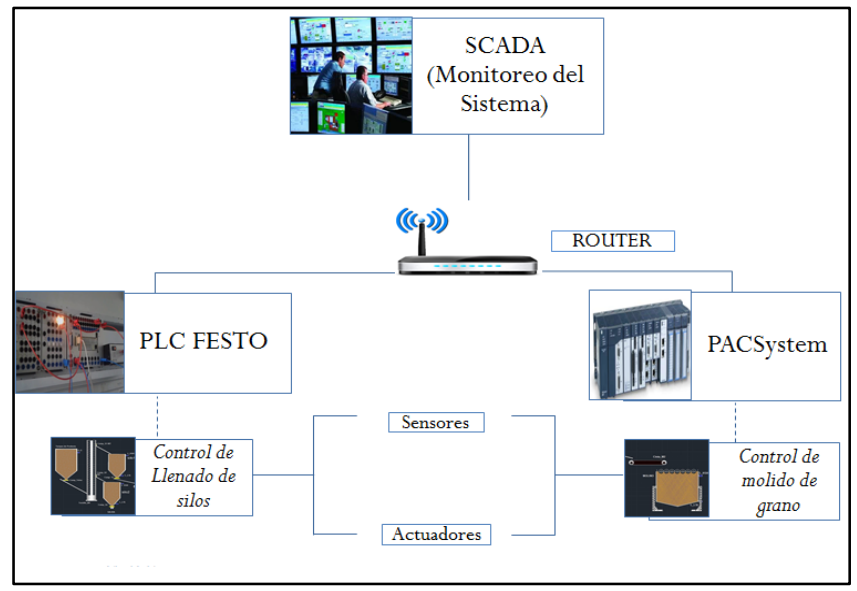

Figura 5. Esquema del diseño del sistema propuesto.

\section{Proceso de control}

Mediante la aplicación de tipo industrial propuesta, para el llenado de silos y molienda de grano, se puede ver reflejada la interacción entre ambos controladores de distinta marca y modelo (PACSystem y Festo Compact), operando en conjunto en una cadena de producción. Cada controlador ejecuta acciones de control para un determinado proceso o subproceso.

El PLC Compact FC34, de la marca Festo, realiza acciones de control para el subproceso del llenado de dos silos contenedores de grano; esta etapa es la parte inicial y el comienzo de la cadena de producción; la secuencia de control que ejecuta es descrita a continuación:

1) Depósito 1 y 2 se encuentran vacíos, se acciona la compuerta de la tolva, se conecta el tornillo sinfín y la compuerta del silo 1, para ser llenados (silo 1 tiene preferencia de llenado).

2) Una vez que el silo 1 se ha llenado, cierra la compuerta de la tolva, deja transcurrir 10 segundos para detener el tornillo sinfín, esto con el propósito de evitar dejar grano remanente dentro del tornillo; trascurrido el tiempo, se detiene el motor del tornillo y cierra la compuerta de entrada del silo 1.

3) Cuando el silo 1 se encuentre lleno, envía una alarma y enseguida se vuelve a abrir la compuerta de la tolva para comenzar a llenar el 
silo 2; la secuencia del proceso se repite al igual que el silo 1.

4) Si ambos silos se encuentran llenos, no se ejecuta alguna secuencia de proceso, hasta que alguno de ambos silos se vacíe.

Por su parte, el PACSystem RX3i, de la marca GE (General Electric), es el encargado de ejecutar el segundo subproceso correspondiente al molido de grano, para su posterior distribución; dentro de su lógica, la secuencia de control que realiza es:

5) El silo 1 es el que suministra grano al molino.

6) Una vez lleno el silo 1, y el depósito del molino se encuentre vacío, se abre la compuerta de salida de este silo, actúa la cinta o banda transportadora, y se acciona el triturador de grano.

7) Una vez lleno el depósito del molino, se cierra la compuerta de salida del silo 1 , y el motor que actúa la cinta se detiene después de 10 segundos para evitar que haya material remanente en la banda; y posteriormente, 5 segundos después, se detiene el motor del molino, enviando una señal de alarma que indica el llenado completo del depósito del molino.

8) En cuanto la compuerta de la tolva se cierra totalmente, transcurren 10 segundos para detener el tornillo sinfín y cerrar la compuerta 1.

Ambos subprocesos cuentan con un selector manual/automático, de manera que cada controlador pueda controlarse así mismo en el momento que se requiera. Asimismo, en la cadena de producción, cuyos subprocesos se encuentran interrelacionados, los controladores intercambian salidas y entradas físicas para poder operar en conjunto.

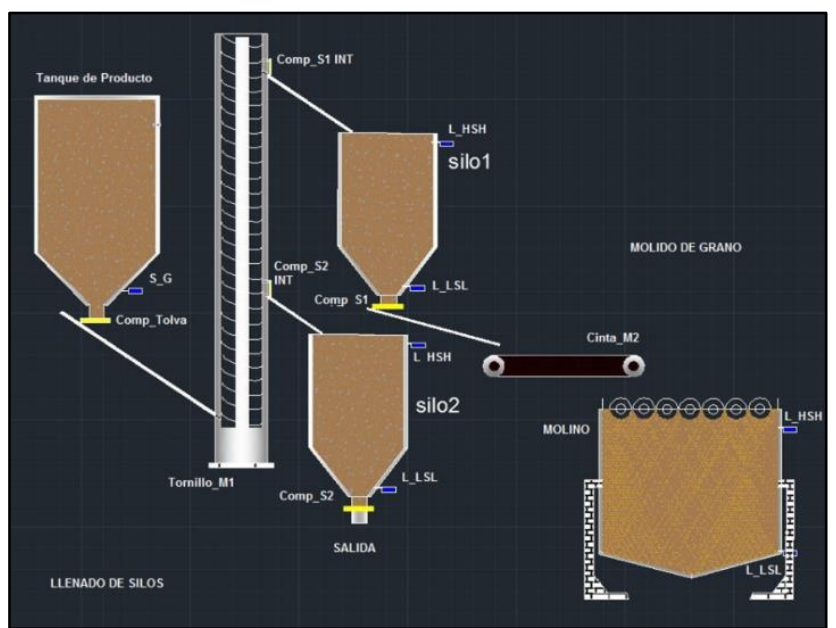

Figura 6. Diseño de aplicación industrial de llenado de silos y molienda de grano (AutoCAD).

\section{Diseño de HMls para el sistema SCADA}

La interfaz gráfica (GUI, por sus siglas en Inglés de Graphical User Interface) tiene como función principal mostrar al operador o usuario la información que se encuentran a nivel de campo; por ejemplo, el estado de una válvula, un motor, niveles en tanques, y otras variables del proceso; todo esto mediante pantallas que permiten monitorear, regular y controlar un determinado proceso. Es la principal herramienta utilizada por operarios y supervisores de línea para coordinar y controlar procesos industriales y de fabricación [10].

El diseño de las interfases de usuario se encuentra dividido en pantallas primarias, secundarias y terciarias; cada una muestra los accionamientos, indicaciones y mediciones de las variables del proceso. Las pantallas primarias muestran el sistema completo, tanto la arquitectura de comunicación como la cadena de producción operando en conjunto.

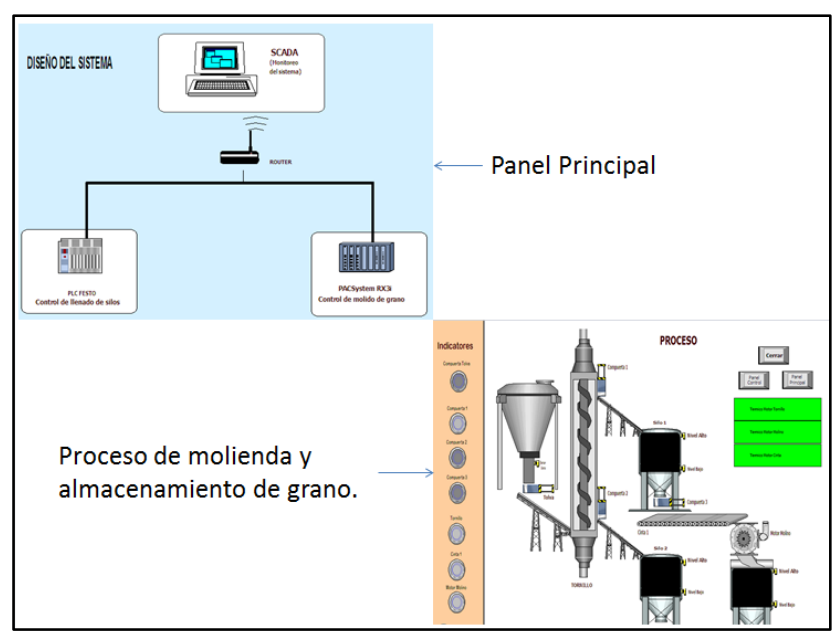

Figura 7. Pantallas primarias de la $\mathrm{HMI}$, realizadas en Wonderware InTouch.

En la actual normativa ANSI-ISA-101.01, la eficacia de una aplicación $\mathrm{HMI}$ se relaciona con la forma en que se estructuran las pantallas. El desempeño global de la operación se ve afectado por la responsabilidad de los operarios, la complejidad del proceso, la presencia de aplicaciones de control avanzado, el sistema de gestión de alarmas, y otros factores adicionales [13].

En las pantallas secundarias, mostradas en la Figura 8, se presentan los subprocesos del sistema tanto para el llenado de los silos, que es llevado a cabo por el PLC Festo Compact FC34, como para el molido de grano, que es ejecutado por el PACSystem RX3i de GE; cada interfaz cuenta con indicaciones y alarmas del proceso que se monitorea.

En las pantallas terciarias, mostradas en la Figura 9, se visualizan los aspectos de los sistemas físicos, tales como los actuadores (motores trifásicos y variadores de 
frecuencia); así mismo, una interfaz que muestra el estado que las variables guardan al momento, los cuales son transferidos a una hoja de datos de la paquetería de Office.

Finalmente, se tiene una interfaz de usuario que permite tomar acciones de control sobre el sistema. El panel de control es manipulado por el operador o usuario encargado del monitoreo del sistema.

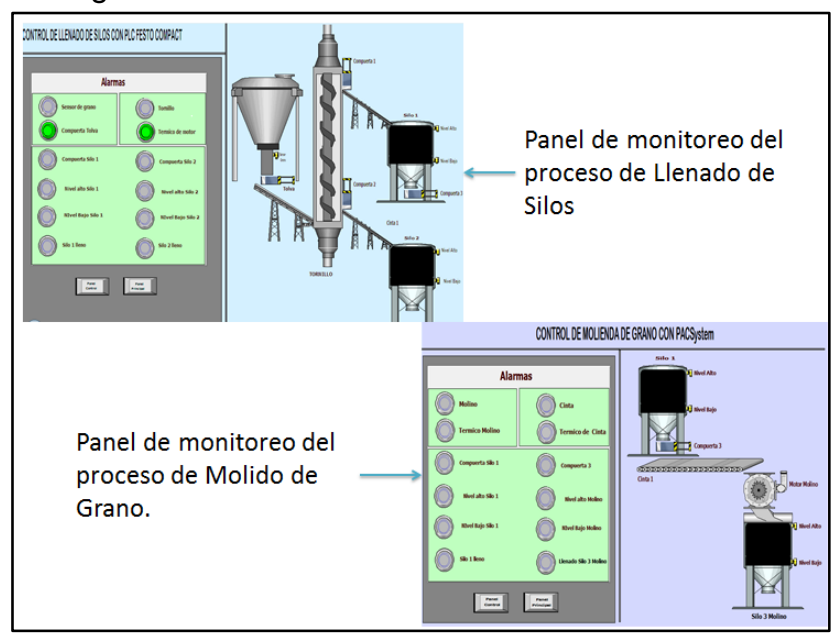

Figura 8. Pantallas secundarias de la HAMI, realizadas en Wonderware InTouch.

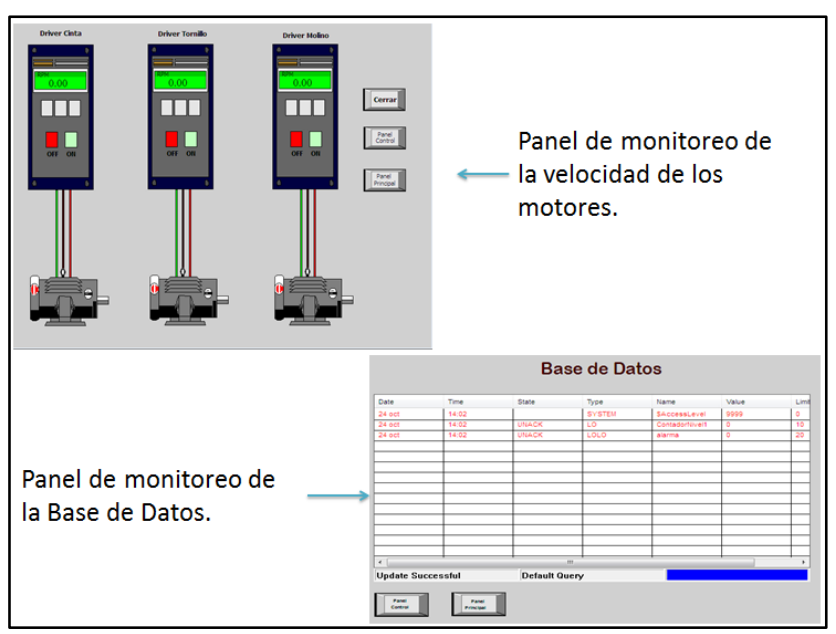

Figura 9. Pantallas terciarias de la $\mathrm{HMI}$, realizadas en Wonderware InTouch.

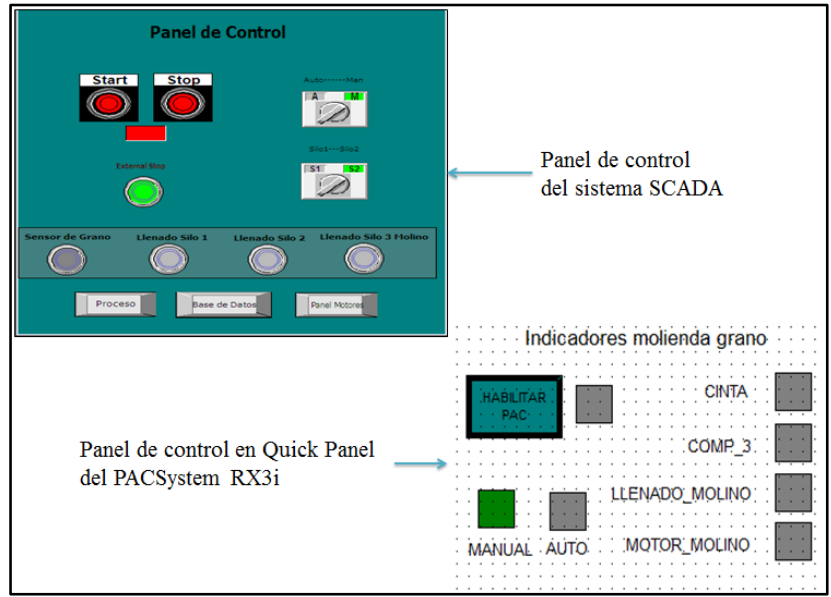

Figura 10. Paneles de control del sistema.

\section{Comunicaciones entre el sistema SCADA Wonderware InTouch y los controladores PACSystem RX3i y PLC Festo Compact FC34}

A continuación, se describe la manera en que fue realizada la comunicación entre el SCADA Wonderware InTouch y el servidor IPC DATA SERVER y el OPC KepServer, para el monitoreo y control del sistema de llenado automatizado de los silos por medio de los controladores PACSystem y el PLC FEC 34. Para realizar la comunicación entre el PLC y el sistema SCADA, existen varios sistemas, como el OPC y el DDE, haciendo uso de ambos en este caso.

Comunicación mediante OPC entre el software SCADA y el PAC

Para establecer la comunicación, primero se debe vincular el software SCADA, en este caso Wonderware InTouch, con el servidor OPC KepServer.

1. En InTouch-Application Manager, el nombre del proyecto creado debe estar visible debajo de la lista de aplicaciones de InTouch. Se selecciona el nombre del proyecto y se accede a File | WindowMaker.

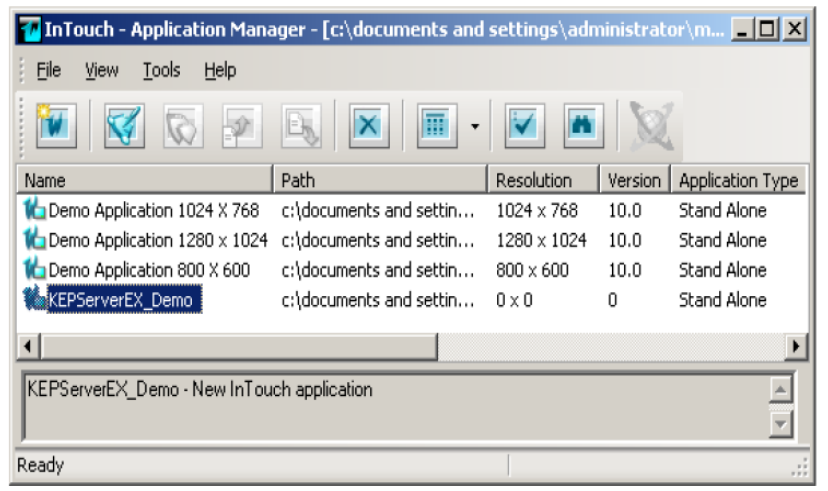

Figura 11. Application Manager InTouch. 
2. En InTouch-WindowMaker, dar click en Special | Access Names.

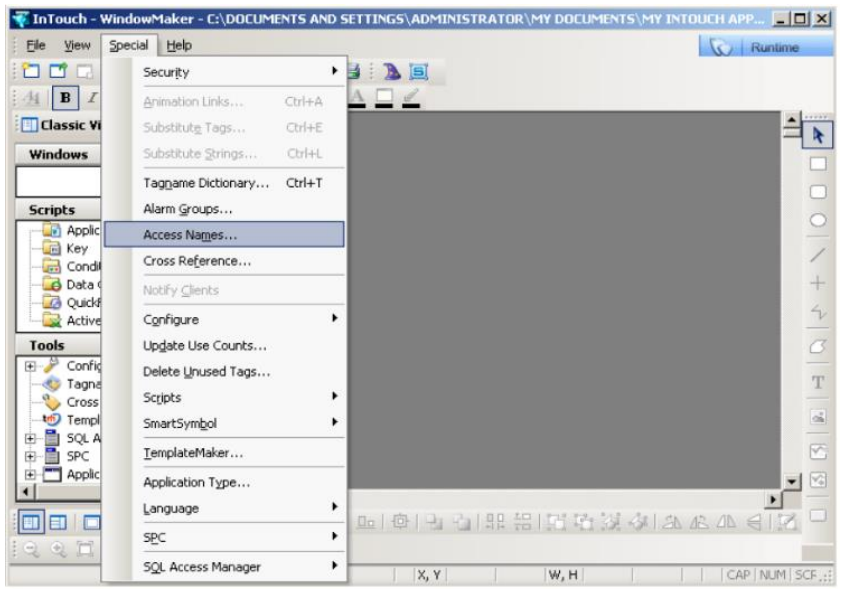

Figura 12. Interfaz de InTouch.

3. Para agregar un nuevo access name, dar click en Add.

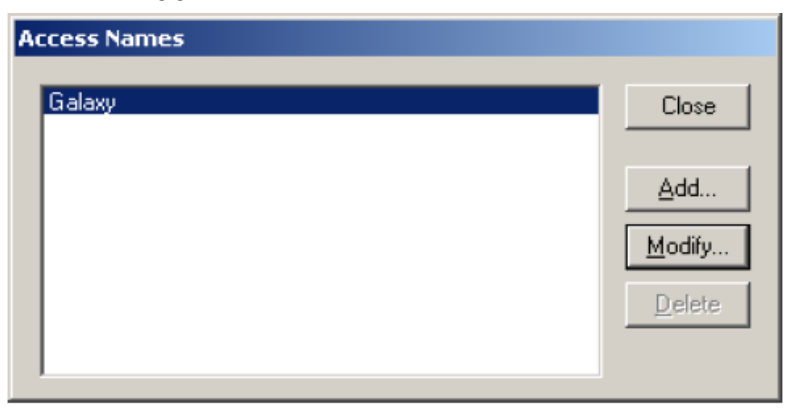

Figura 13. Configuración del Acces Name.

4. En agregar Access Name, realizar los siguientes cambios.

\begin{tabular}{|l|l|}
\hline Modify Access Name & \\
\hline Access PAC1 \\
Node Name:
\end{tabular}

Figura 14. Configuración del Acces Name.

- En Access, ingresar un nombre de acceso único. En este tutorial se utiliza "PAC1".

- $\quad$ En nombre de nodo (Node Name), dejar el campo en blanco.
- $\quad$ En nombre de la aplicación (Application Name), conservar la entrada predeterminada: "server_runtime".

- En nombre de tema (Topic Name), escribir el primer alias que se creó anteriormente; en este caso Channel1_PAC.

- En Cuál protocolo utilizar (Which protocol to use), seleccionar DDE.

5. Al terminar, dar click en Aceptar (OK).

Posteriormente, para establecer la comunicación entre el PAC y el OPC, que ya fue enlazado al SCADA, se debe efectuar lo siguiente.

1. Dar click en "Click to add a channel" y asignar un nombre al canal; en este caso se nombró PAC.

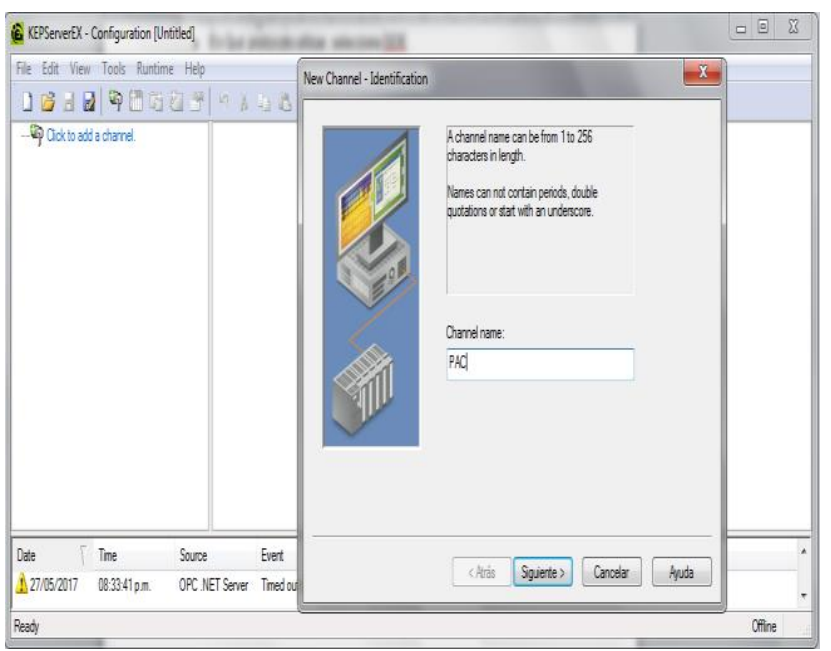

Figura 15. Configurando un Canal de comunicación en KepServer.

2. Ahora dar click en "next" y seleccionar el tipo de hardware en GE Ethernet. 


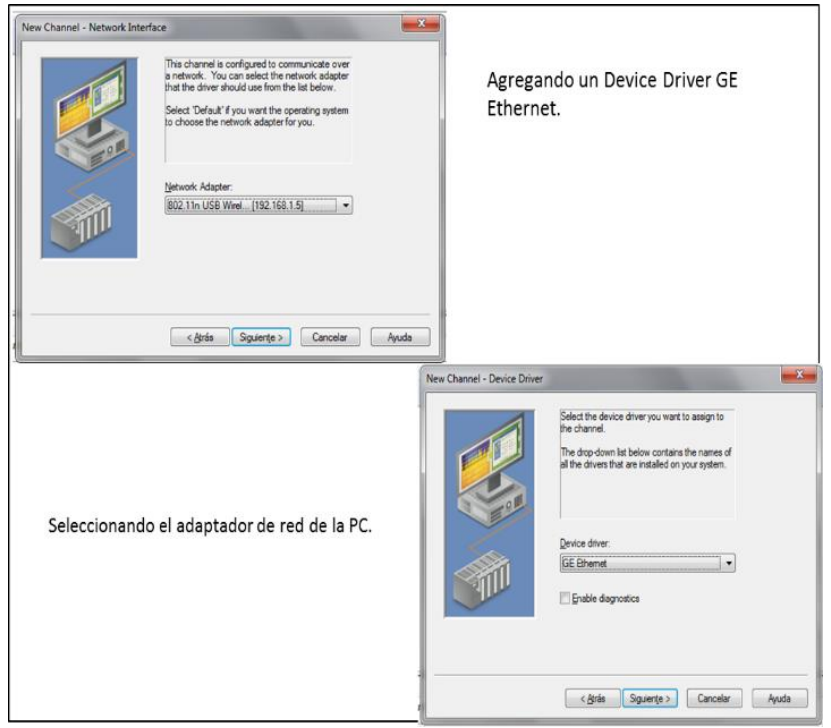

Figura 16. Configurado Hardware.

3. Dar click en next en repetidas ocasiones, sin realizar cambios, hasta finalizar y que aparezca la siguiente ventana (Figura 17); entonces dar click en "Click to add a device".

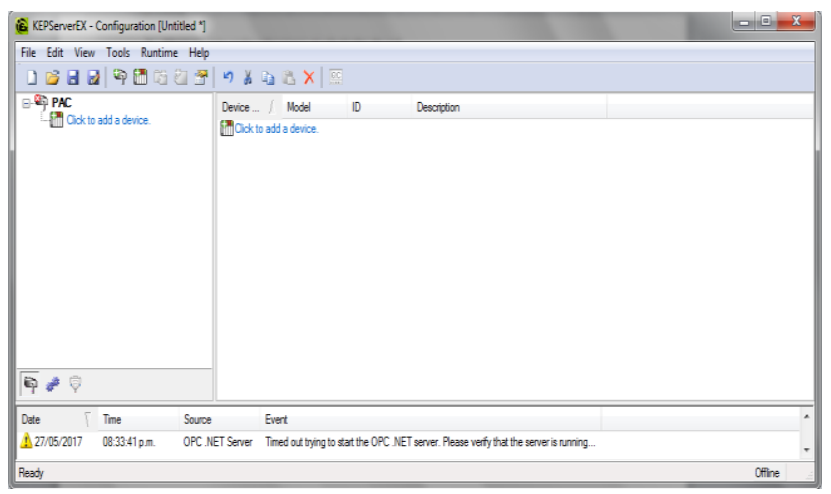

Figura 17. Configuración de un nuevo dispositivo.

4. Asignar un nombre a dispositivo en Device name; en este caso "PAC".

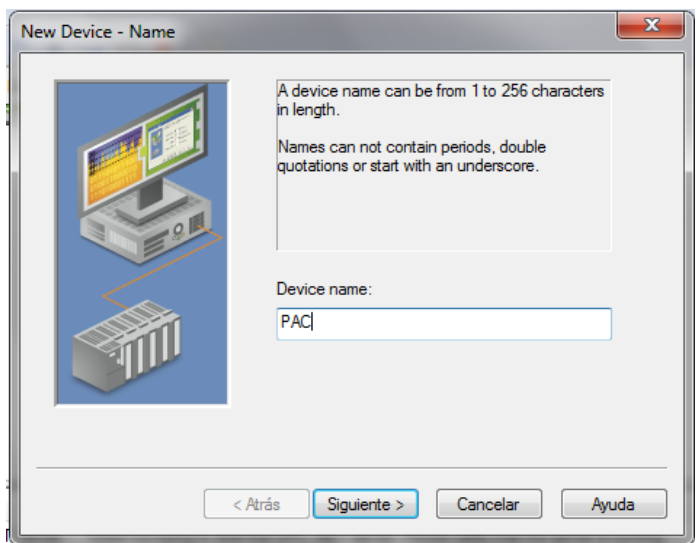

Figura 18. Configuración de un nuevo dispositivo.

5. Seleccionar el modelo de dispositivo, en este caso "PACSystems"

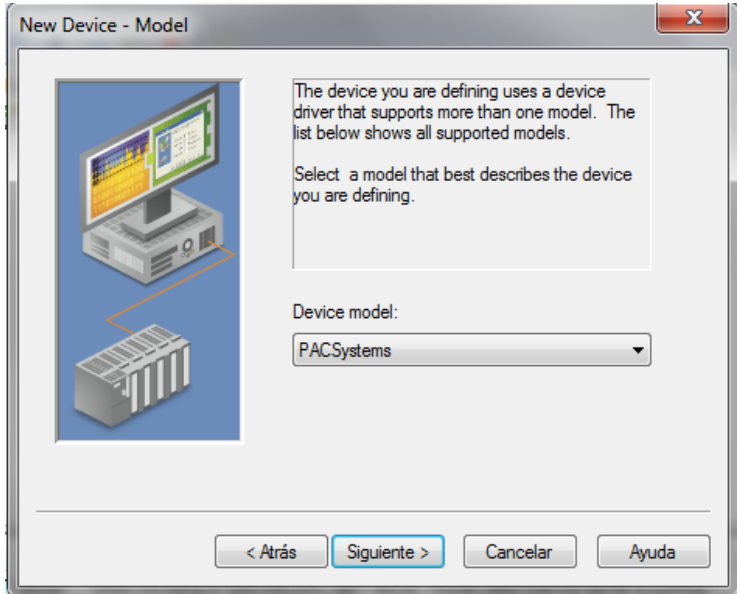

Figura 19. Configuración de un nuevo dispositivo (continuación).

\section{Agregar dirección IP o “Device ID” 10.0.0.10}

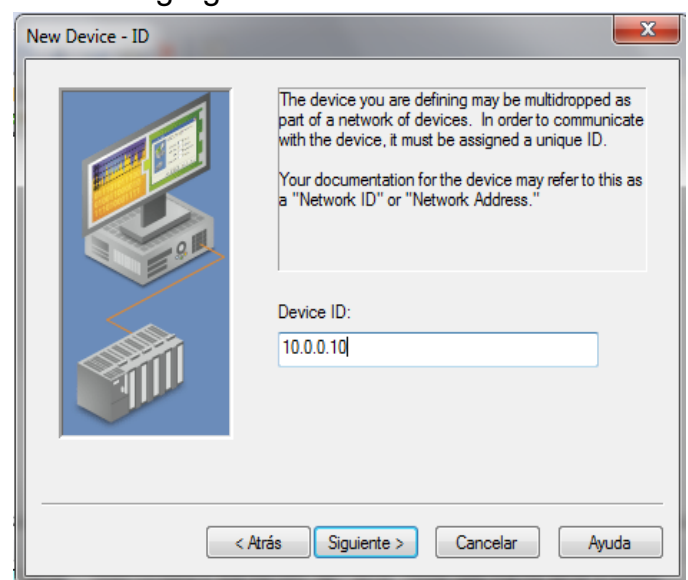

Figura 20. Configuración de un nuevo dispositivo (continuación).

7. Dar click en next en repetidas ocasiones, sin modificar ningún parámetro, hasta finalizar y se muestre la siguiente ventana.

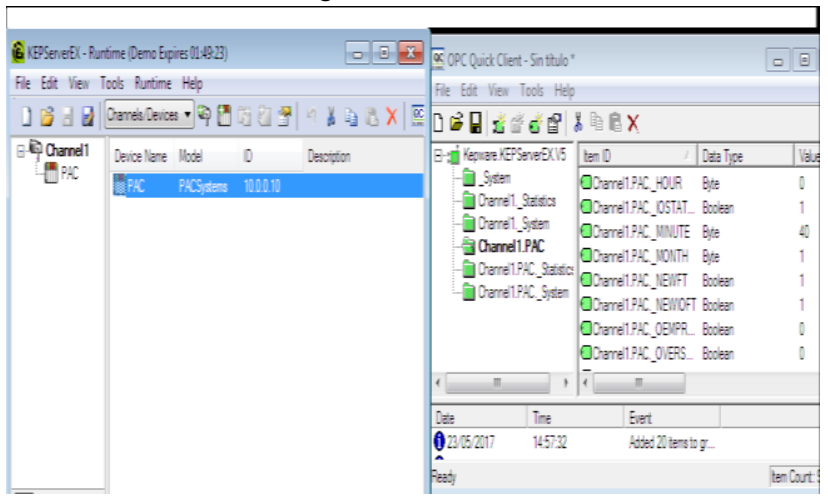

Figura 21. Configuración de un nuevo dispositivo (continuación).

8. Por último, para habilitar la comunicación entre OPC y PAC, se carga el algoritmo de control mediante Proficy Machine Edition, y en el OPC 
dar click en el botón "Quick Client", con lo cual aparecerá otra ventana donde se puede observar el estado de las variables del PAC.

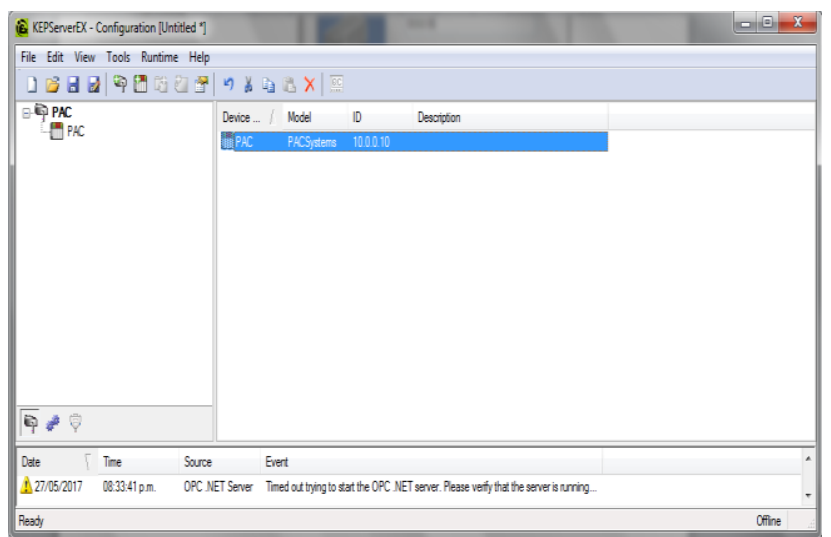

Figura 22. Habilitación de la comunicación entre el OPC y el PAC.

\section{Comunicación DDE entre el SCADA Wonderware InTouch y el servidor de Festo IPC DATA SERVER}

Configuración del servidor IPC DATA SERVER de Festo Con el fin de establecer la comunicación entre el PLC y la PC, se ejecuta la aplicación denominada IPC Data Server de Festo, y se configura la dirección IP, lo cual permite un intercambio dinámico de datos (DDE) entre el sistema de control controlado por el PLC y la PC donde reside el sistema SCADA, tal como se muestra en la Figura 23.

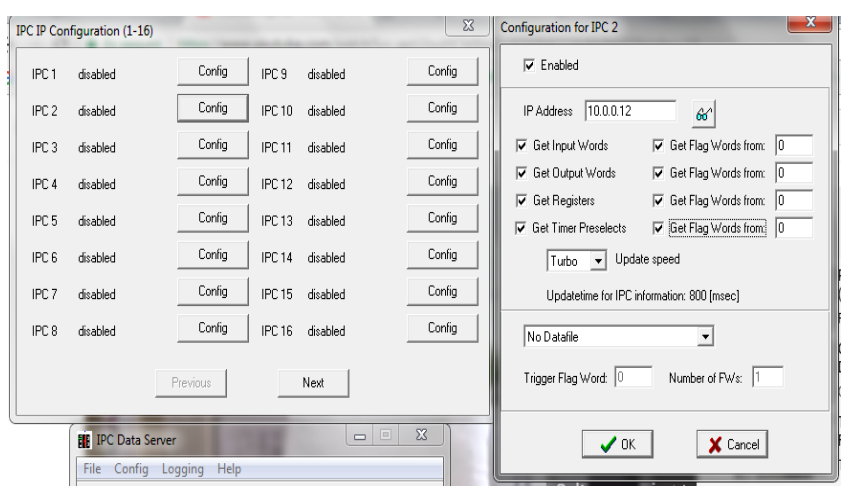

Figura 23. Configuración del IPC Data Server de Festo.

En este caso se configuró el canal IPC para el PLC, colocando su IP respectiva.

Configuración del SCADA InTouch para la comunicación con el servidor.

Una vez creada la HMI en InTouch, se procede ahí mismo a configurar el Access Name para enlazar datos vía DDE desde otras aplicaciones Windows hacia InTouch, como se aprecia en la Figura 24.

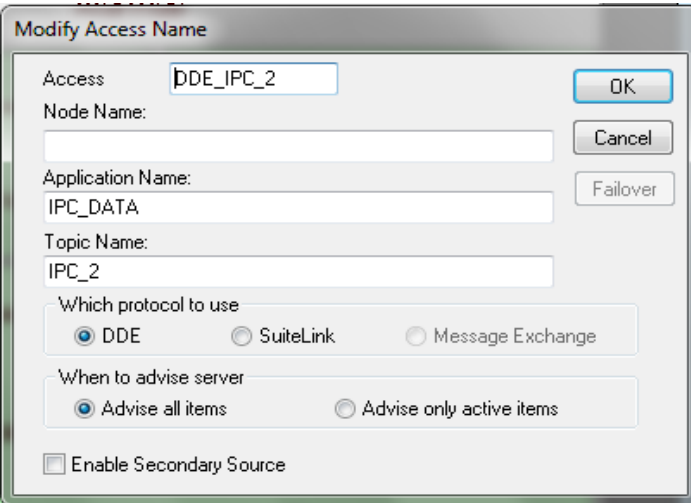

Figura 24. Configuración del Access Name en InTouch.

Direccionamiento de variables en los Tagname de InTouch con las variables del PLC Festo

El direccionamiento de las variables en el software de programación de PLCs FST de Festo, se realiza de la siguiente manera:

- $\quad$ Entradas Físicas: 10.0, 10.1, 10.2, $\ldots$, sucesivamente

- $\quad$ Salidas Físicas: 00.1, 00.2, 00.3,...

- Memorias (Flag Words): F0.1, F0.2, $\mathrm{F} 0.3, \ldots$

- $\quad$ Registros: R1, R2, R3,...

Por su parte, en el SCADA Wonderware InTouch el direccionamiento de las variables, en especial de las entradas, salidas y memorias, se realiza de forma distinta, como se muestra en los ejemplos de a continuación.

\section{Item/Point Naming Examples}

Los siguientes ejemplos muestran la validación de los nombres de las unidades [12].

- $\quad$ M12.0 - Flag bit 0 in Flag word 12;

- $\quad$ E5.14 - Input bit 14 in Input word 5;

- $\quad$ A0.0 - Output word 0, unsigned;

- $\quad$ T22 - Timer number 22;

- R256 - Operand address out of range;

\section{Configuración del Access Name}

Desde la opción Access Name de InTouch, se configuran las señales de entrada y salida que posea el sistema, teniendo en cuenta si son continuas o discretas. Esta acción implica el uso de Tagnames. 


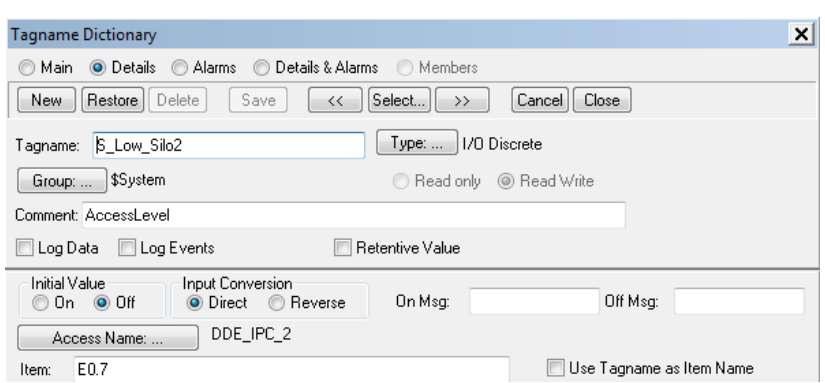

Figura 25. Configuración de Tagnames desde InTouch.

\section{Algoritmo de control del PACSystem}

El subproceso de molienda de grano es controlado mediante un PAC de GE. El algoritmo de control fue desarrollado y programado a través del software ProficyMachine Edition, propio de GE. La programacion de esa lógica fue realizada en el lenguaje denominado Diagrama Escalera (LD por sus siglas en Inglés de Ladder Diagram), uno de los lenguajes normados por la IEC 61131-3. La función principal de la logica de control es hacer que el proceso sea controlado automaticamente; las acciones que han de ejecutarse para ello han sido programadas en el controlador y guardadas 0 almacenadas en su memoria interna.

Para el control de los motores que representan el accionamiento de la banda, el molino y el tornillo, su arranque se realiza por medio de la bobina M2 (Bobina de Arranque) (en el código en LD, Figura 26); asimismo, en serie se encuentran dos contactos llamados "Msilo1" (memoria de silo 1, asociado a memoria de PLC Festo) y "TimerBanda", cuya función es permitir la activación de las líneas correspondientes a los contactos asociados para activar las bobinas "MBanda" y "Molino" (salidas físicas del sistema). En la programación, éstas bobinas alimentan a las líneas 5 y 7 , respectivamente; el propósito de esta lógica planteada es que al momento de enviar la señal al Variador de Frecuencia, es necesario activar dos de sus terminales de control; por lo que, al activarse una de las salidas físicas, es necesario activar otra para accionar un motor en su primera velocidad.

Para las líneas 9 y 10 , se tiene un contacto NA (Normalmente Abierto), llamado "TornilloFesto", asociado a una salida física del PLC Festo; el contacto recibe la señal y activa la bobina "Tornillo" (salida física del sistema); de igual manera, la misma bobina alimenta la línea siguiente para activar la bobina "JTornillo" y hacer funcionar al motor una vez que se envía la señal.

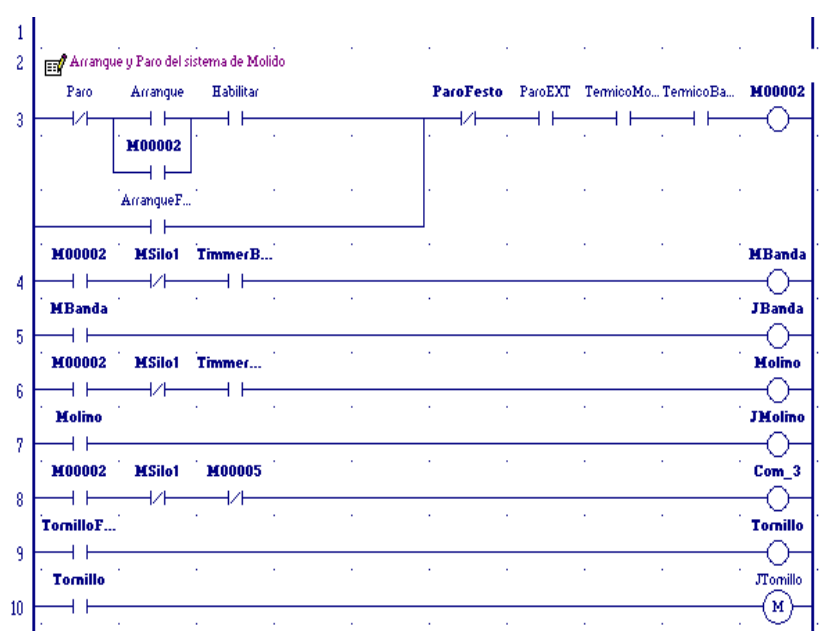

Figura 26. Arranque y paro del sistema de molido.

\section{Algoritmo de control del Festo Compac}

El algoritmo de control alojado en el PLC Festo se encarga del control del llenado de los 2 silos contenedores, por medio del control de la apertura de las compuertas que les abastecen el grano, por medio de cilindros neumáticos así como de sensores de nivel, bajo y alto, en cada silo.

Primeramente, se tiene un arranque/paro del sistema completo, así como un selector de control manual/automático para el llenado de los silos. Cabe mencionar que también es posible realizar el arranque del sistema desde el PACSystem RX3i, ya que ambos controladores se encuentran comunicados y enlazados por medio del software SCADA Wonderware Intouch y la red Ethernet.

A continuación, se muestra de manera resumida la lógica de control para el arranque del sistema, con las condiciones de que (1) el Tanque principal tenga grano para que pueda alimentar al tornillo (sinfín) de distribución que llena tanto al Silo 1 como al Silo 2, y (2) el sensor de nivel del Silo 1 debe detectar siempre nivel bajo. Esto se logra por medio de los sensores digitales que detectan presencia de grano en los tanques, causando que una compuerta se abra y abastezca al tornillo.

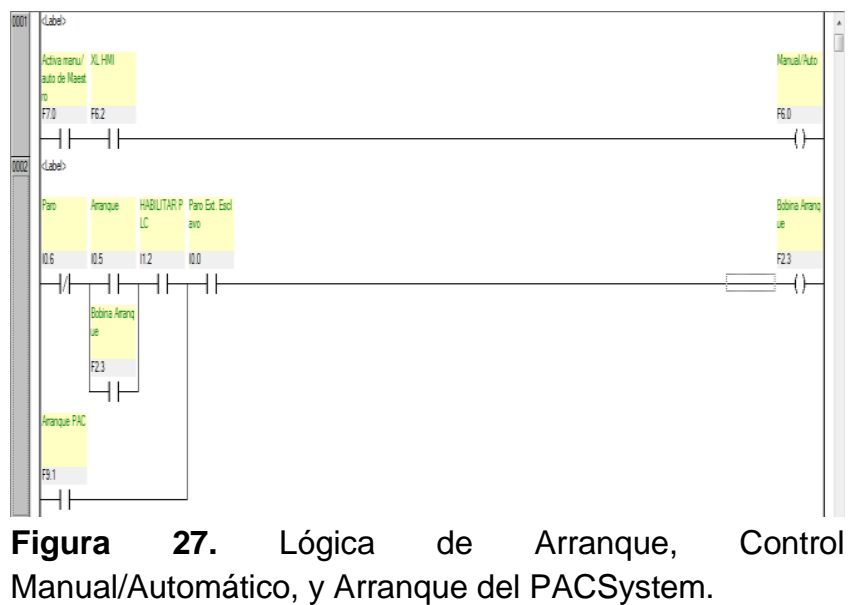




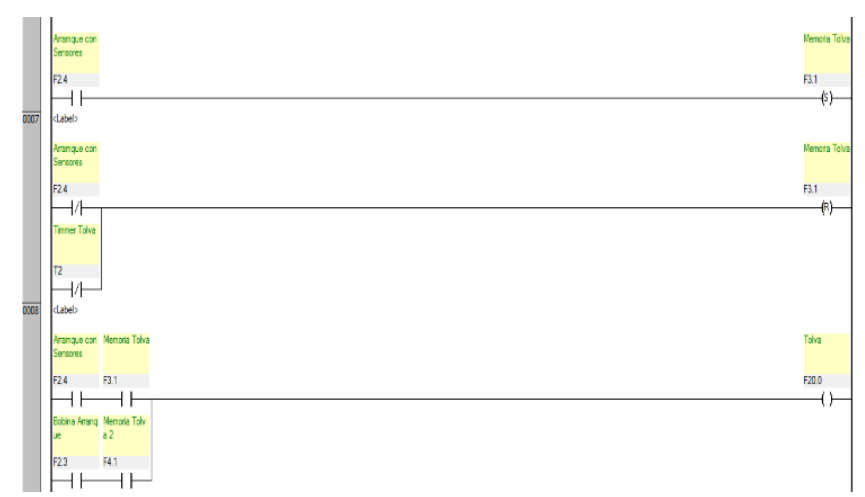

Figura 28. Control de apertura de la tolva.

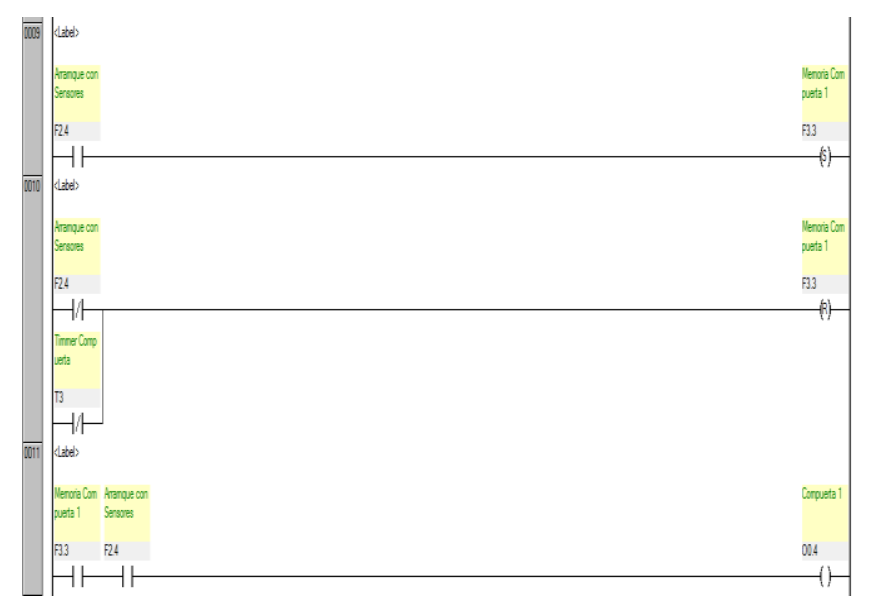

Figura 29. Control de apertura de la Compuerta 1.

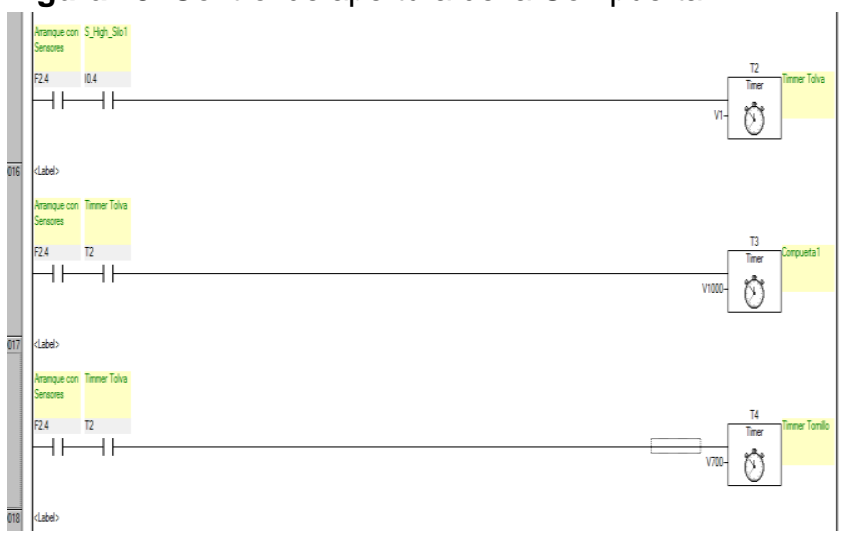

Figura 30. Timmers para el control del apagado de los actuadores.

\section{Configuración de los Drivers para el control de los motores empleados}

Para realizar el control de motores con el PACSystem, se emplearon variadores de frecuencia (drivers) de la marca GE modelo AF-60; robustos modelos trifásicos de 230 VCA, equipados con controles de freno, sistema de realimentación $\mathrm{PI}$, comunicación RS-485 por protocolo MODBUS RTU, control digital y analógico [14].

En el caso que se estudia en el presente trabajo, se ha empleado el control digital, configurando la primera de siete velocidades en 520 rpm (revoluciones por minuto), que representa el $30 \%$ de $1730 \mathrm{rpm}$ que es la velocidad nominal del motor. Para el cableado se emplearon 2 bobinas del driver que representan el arranque y la $1^{\text {a }}$ velocidad, y se conectaron como especifica la tabla de conexiones del driver.

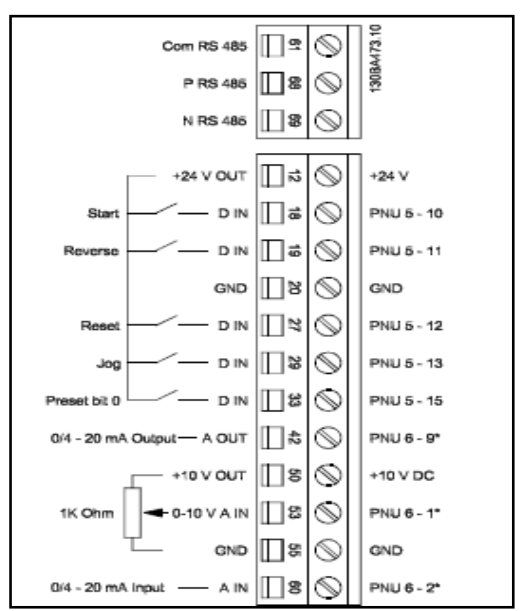

Figura 31. Vista general para las conexiones de las terminales de control [15].

Dentro de la configuración del driver, se toman las siguientes consideraciones en cuanto a los datos del motor.

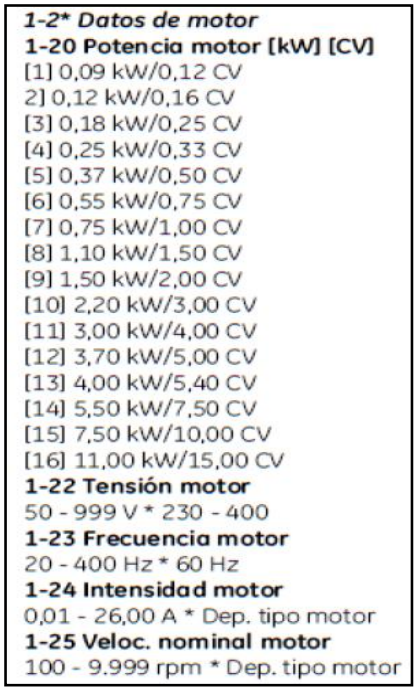

Figura 32. Datos del motor [16]

\section{Equipo neumático empleado para el sistema de control}

Para representar el accionamiento de las compuertas de las tolvas, que llenan o vacían los silos, se emplearon válvulas y pistones neumáticos del Equipo Neumático Festo TP101, de Festo Didactic, el cual es un sistema de propósitos didácticos en materia de sistemas y técnicas de automatización industrial que se rige por diversos planes de estudios y exigencias que plantean las profesiones correspondientes [17]. Los equipos didácticos están clasificados según los siguientes criterios: 


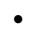

Equipos didácticos básicos, para la adquisición de conocimientos tecnológicos básicos generales.

- $\quad$ Equipos didácticos tecnológicos, que abordan temas de importancia sobre la técnica de control y regulación.

- $\quad$ Equipos didácticos de funciones, que explican las funciones básicas de sistemas automatizados.

- $\quad$ Equipos didácticos de aplicaciones, que permiten estudiar en circunstancias que corresponden a la realidad práctica.

Los equipos didácticos abordan temas técnicos tales como: neumática, electroneumática, controladores lógicos programables, automatización con computadoras personales $(\mathrm{PC})$, hidráulica, electrohidráulica, hidráulica proporcional, y técnicas de manipulación.

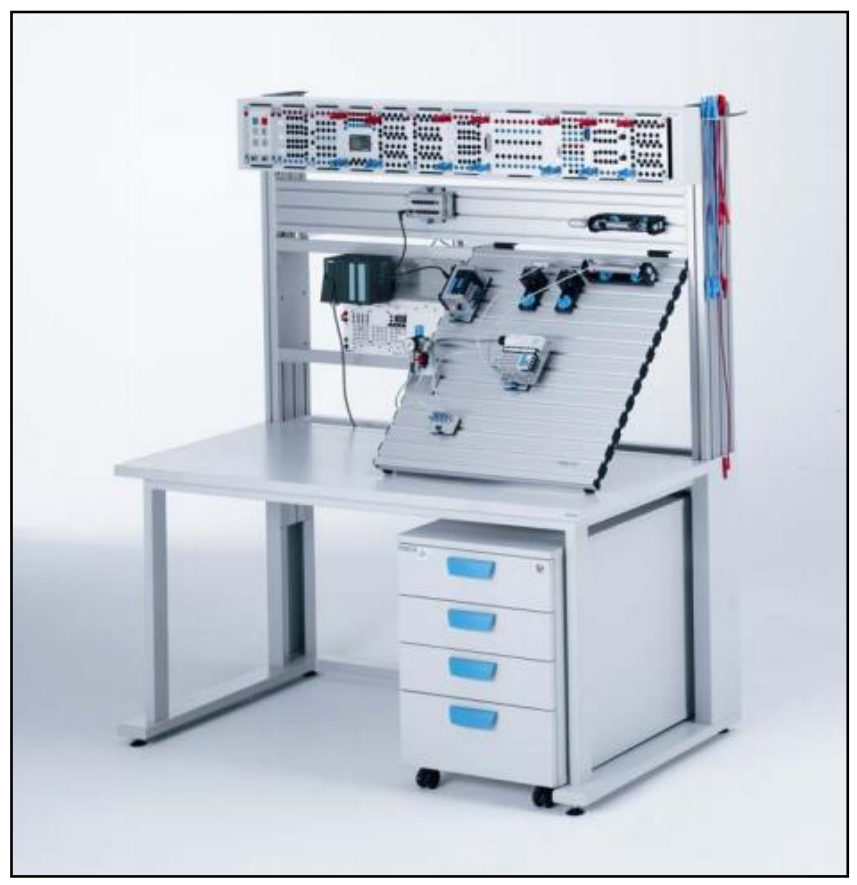

Figura 33. Equipo Neumático Festo TP101 [17].

\section{Controladores empleados}

Para el subproceso del llenado de silos, se empleó el PLC Festo Compact FC34, el cual posee las siguientes características.

- $\quad$ Potente procesador AMD: AM186 (20 $\mathrm{MHz}, 2$ Mips) con acceso de memoria de 16 bits, 512 Kbytes de RAM.

Disco flash: Aproximadamente 100 Kbytes gratis

- Ethernet 10BASE T: Telnet, TFTP, TCP/IP, Servidor Web

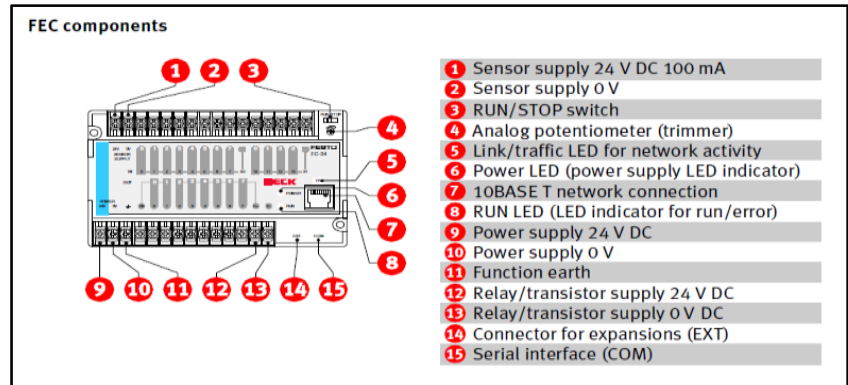

Figura 34. Partes del PLC FEC34 Festo [18].

Para la etapa del molino de grano, se empleó el PACSystem RX3i de la marca GE, el cual es un poderoso e innovador Controlador de Automatización Programable, PAC, en la familia PACSystems. El RX3i cuenta con un único motor de control y un entorno de programación universal que facilita la portabilidad de aplicaciones a través de múltiples plataformas de hardware y ofrece una elevada compatibilidad de control [19].

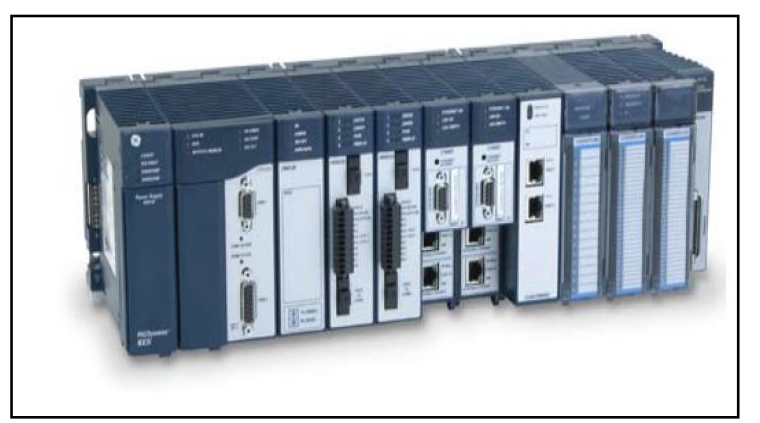

Figura 35. PACSystem RX3i de General Electric [19].

\section{Características del PACSystem RX3i}

- $\quad$ Procesador de alta velocidad.

- Apoyo bus posterior Dual por ranura del módulo.

- Plano posterior de serie, para facilitar la migración de las Series 90-30 I/O existentes.

- Ofertas de CPU múltiples que cumplen varios criterios de rendimiento, hasta e incluyendo un procesador Intel $\mathrm{CPU}$ de $1 \mathrm{GHz}$ para la programación avanzada y rendimiento con 64 Mbytes de memoria.

- Apoyo a las comunicaciones abiertas que incluye Ethernet, Genio, Profibus, HART, DeviceNet y en serie.

- Soporta módulos de E/S discretas, analógicas universales aislado, analógicos, analógicos de alta densidad, de contador de alta velocidad, y de movimiento de alta densidad.

\section{Conclusiones}

La interconexión de controladores de distinta marca para su interacción para el desarrollo de procesos industriales 
en cadena es de suma importancia y necesidad, ya que en las plantas, en numerosas ocasiones, se emplean controladores de distintas firmas o proveedores, lo que demanda la labor de un intermediario experto que logre esa interacción entre los subprocesos. En este trabajo, haciendo uso del software InTouch de tipo supervisorio, se estableció una comunicación e interrelación entre ambos equipos controladores empleados, donde se ha estimado que el tiempo de respuesta de los instrumentos de campo así como las HMI y sus animaciones no sobrepasa los $50 \mathrm{~ms}$ (milisegundos), por lo que se considera que se tiene un proceso suficientemente rápido en envío y recepción de datos; además de la generación de una base datos la cual genera un histórico que puede ser exportado a una hoja de datos que provee la paquetería de Office. Finalmente, el beneficio que propone y plantea el proyecto es la posibilidad de poder expandir el uso de más controladores, con el fin de expandir la planta incrementando subprocesos.

\section{Referencias}

[1] I. S. I. 7498-1, Open Systems Interconnection - Basic Reference Model, Segunda edición ed., 1994, p. 59.

[2] A. R. Penin, «Sistemas SCADA,» de El Sistema SCADA, 2da Edición ed., Barcelona, Barcelona: MARCOMBO, S.A., 2007, p. 447.

[3] A. Creus, « $8^{a}$ EDICIÓN INSTRUMENTACIÓN INDUSTRIAL,» Octava Edición ed., Barcelona, España, Barcelona: Alfaomega Grupo Editor, S.A. de C.V., México, Septiembre 2010, p. 792.

[4] W. A. Daneels, «WHAT IS SCADA?,» de International Conference on Accelerator and Large Experimental Physics Control Systems, Trieste, Italy, 1999.

[5] J. A. V. Costa, «Computer integrated CIM».

[6] M. OPC, «Matrikon OPC,» Matrikon , 2017. [En línea]. Available: https://www.matrikonopc.com/downloads/572/webcasts/index.aspx. [Último acceso: 11 Septiembre 2017].

[7] V. GUERRERO y R. L. Y. LUIS MARTINEZ, COMUNICACIONES INDUSTRIALES, S.A. MARCOMBO, 2010, pp. 255-256.

[8] E. A. B. L. A. Bryan, PROGRAMMABLE CONTROLLERS, THEORY AND IMPLEMENTATION, Second Edition ed., Atlanta, Georgia: Published by Industrial Text Company, 1997, p. 1025.

[9] I. International Electrotechnical Commission, «IEC 61131-1, Programmable controllers - General information,» 2003.

[10] Wonderware, «Wonderware, Interfaz Hombre-Máquina (HMI), 2017. [En línea]. Available: http://www.wonderware.es/hmiscada/que-es-hmi/. [Último acceso: 10 Septiembre 2017].

[11] K. KepServe, «KepServer User's Manual,» 2017. [En línea]. Available: https://www.kepware.com/enus/products/kepserverex/documents/kepserverex-manual/. [Último acceso: 17 Septiembre 2017].
[12] K. AUTOMATION, FESTO IPC Communication Server for Microsoft Windows and Intouch Applications, Helsinki, Finlandia: KLINKMANN, 2013, pp. 1-2.

[13] A. N. STANDARD, «ANSI/ISA-101.01-2015, Human Machine Interfaces for Process Automation Systems,» 2015, p. 59.

[14] G. Industrial, «AF-60 LP* Micro Drives,» General Electric, [En línea]. Available: http://www.geindustrial.com/products/drives/af-60lp-micro-drives. [Último acceso: 259 2017].

[15] C. \&. I. GE, «AF-60 LPTM Micro Drive».

[16] G. C. \&. Industrial, «AF-60 LP Micro Drive».

[17] Festo, «Neumática Nivel Basico,» 2005. [En línea]. Available: http://www.festo-didactic.com/. [Último acceso: 17 Septiembre 2017].

[18] B. Festo, «LabElectronica,» [En línea]. Available: http://labelectronica.weebly.com/uploads/8/1/9/2/8192835/fec_fc34.pd f. [Último acceso: 14 Septiembre 2017].

[19] G. Automation, «GE Automation,» [En línea]. Available: http://www.geautomation.com/products/pacsystems-rx3i-controller. [Último acceso: 14 Septiembre 2017].Dilla T, Valladares A, Lizán L, Sacristán JA. Adherencia y persistencia terapéutica: causas, consecuencias y estrategias de mejora. Aten. Primaria 2009; 41(6): 34248.

[2] Silva G, Galeano E, Correa J. Compliance with the treatment. Acta Médica Colombiana 2005; 30(4): 268-73.

[3] Viesca TC. Paternalismo médico y consentimiento informado. En: Márquez-Romero R, Rocha- Cacho WV, editors. Consentimiento informado. Fundamentos y problemas de su aplicación práctica. 1st ed. México: UNAM; 2017: 1-22.

[4] Grady P, Lucio-Gough L. El automanejo de las enfermedades crónicas: un método integral de atención. Panam. J. Public. Health. 2015; 35(9): 187-194.

[5] Cañete-Villafranca R, Guilhem D, Brito-Pérez K. Paternalismo médico. Rev. Med. Elect. 2013; 35(2): 144-152.

[6] Caballo C, Pascual A. Entrenamiento en habilidades de automanejo en personas con enfermedades crónicas: un estudio cuasi-experimental. En: Universidad de Salamanca, editor. IX Jornadas Científicas Internacionales de investigación sobre personas con discapacidad. Salamanca; INICO; 2012: 1-12.

[7] Millaruelo-Trillo JM. Importancia de la implicación del paciente en el autocontrol de su enfermedad: paciente experto. Importancia de las nuevas tecnologías como soporte al paciente autónomo. Aten. Primaria 2010; 42(1): 41-47.

[8] Bonal-Ruiz R, Cascaret-Soto X. Reflexión y debate ¿Automanejo, autocuidado o autocontrol en enfermedades crónicas? Acercamiento a su análisis e interpretación. MEDISAN. 2009; 13(1): 1-10.

[9] Lorig K, Ritter PL, Villa F, Piette JD. Spanish diabetes self-management with and without automated telephone reinforcement. Diabetes Care 2008; 31(3): 408-14.

[10] Kidney Disease Improved Global Outcomes (KDIGO). KDIGO 2012 Clinical Practice Guideline for the Evaluation and Management of Chronic Kidney Disease. Off. J. Int. Soc. Nephrol. 2013; 3(1): 1-163. 